\title{
Monocular Pupillometry
}

National Cancer Institute

\section{Source}

National Cancer Institute. Monocular Pupillometry. NCI Thesaurus. Code C120700.

A technique used to measure the diameter of a single pupil in response to stimuli. 